\title{
Mortality difference between early-identified sepsis and late-identified sepsis
}

\author{
Woon Jee', Sion Jo ${ }^{2,3,4}$, Jae Baek Lee ${ }^{2,3}$, Youngho Jin ${ }^{2,3}$, Taeoh Jeong ${ }^{2,3}$, \\ Jae Chol Yoon ${ }^{2,3}$, Boyoung Park ${ }^{4}$
}

'Department of Emergency Medicine, Chonbuk National University Hospital, Jeonju, Korea ${ }^{2}$ Department of Emergency Medicine, Chonbuk National University Medical School, Jeonju, Korea ${ }^{3}$ Research Institute of Clinical Medicine of Chonbuk National University and Biomedical Research Institute of Chonbuk National University Hospital, Jeonju, Korea

${ }^{4}$ Department of Medicine, Hanyang University College of Medicine, Seoul, Korea

Objective The aim of the study was to compare the mortality rates of patients with early-identified (EI) sepsis and late-identified (LI) sepsis.

Methods We performed a retrospective chart review of patients admitted to the emergency department and diagnosed with sepsis. El sepsis was defined as patients with a Sequential Organ Failure Assessment (SOFA) score $\geq 2$, based on 3 parameters of the SOFA score (Glasgow coma scale, mean arterial pressure, and partial pressure of oxygen/fraction of inspired oxygen ratio), measured within an hour of emergency department admission. The remaining patients were defined as LI sepsis. The primary outcome was in-hospital mortality.

Results Of the total 204 patients with sepsis, 113 (55.4\%) had El sepsis. Overall mortality rate was $15.7 \%$, and El sepsis group had significantly higher mortality than $\mathrm{LI}$ sepsis (23.0\% vs. 6.6\%, $\mathrm{P}=0.003)$. The patients with El sepsis, compared to those with $\mathrm{LI}$ sepsis, had higher SOFA score (median: 4 vs. 2, P<0.001); Acute Physiology and Chronic Health Evaluation (APACHE) II score (median: 14 vs. 10, $\mathrm{P}<0.001$ ); were more likely to progress to septic shock within 6 hours after admission (17.7\% vs. 1.1\%, $\mathrm{P}<0.001)$; were more likely to be admitted to the intensive care unit (2.2\% vs. $1.1 \%, P=0.001)$.

Conclusion Mortality was significantly higher in the El sepsis group than in the LI sepsis group.

Keywords Sepsis; Mortality; Shock, septic

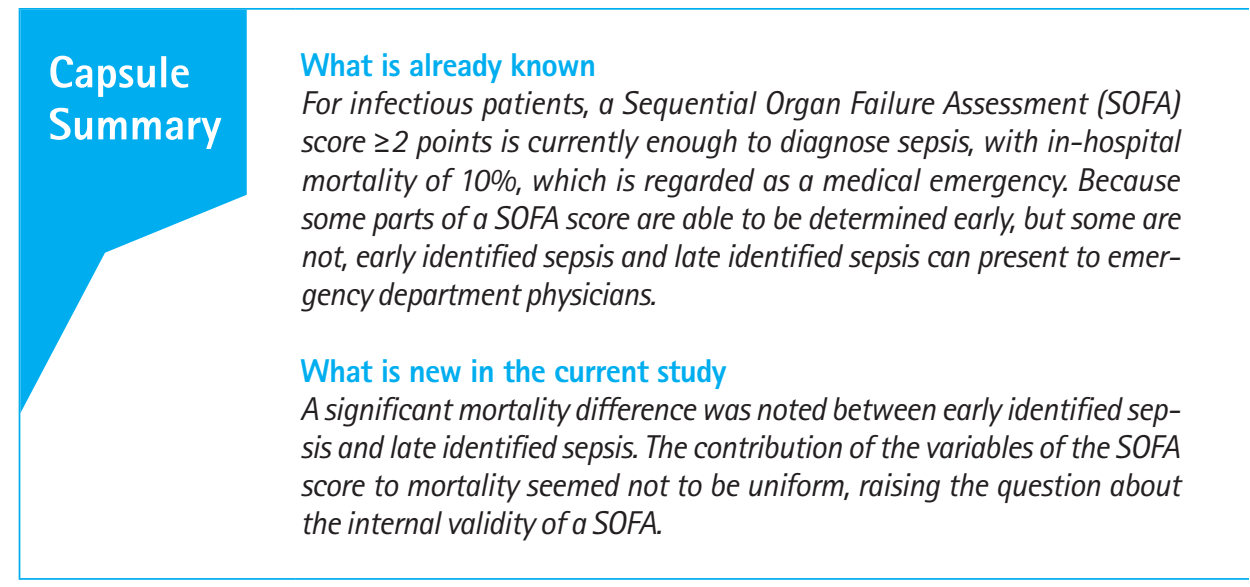

Received: 1 February 2019

Revised: 25 March 2019

Accepted: 3 April 2019

Correspondence to: Sion Jo Department of Emergency Medicine, Chonbuk National University, 567 Baekje-daero, Deokjin-gu, Jeonju 54907, Korea

E-mail: sionjo@jbnu.ac.kr ORCID

http://orcid.org/0000-0001-7667-0367

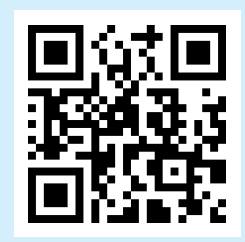

How to cite this article:

Jee $W_{\text {, Jo }}$, Lee JB, Jin Y, Jeong T, Yoon JC, Park B. Mortality difference between early-identified sepsis and late-identified sepsis. Clin Exp Emerg Med 2020;7(3):150160. https://doi.org/10.15441/ceem.19.009

This is an Open Access article distributed under the terms of the Creative Commons Attribution Non-Commercial License (https:// creativecommons.org/licenses/by-nc/4.0/). 


\section{INTRODUCTION}

In 2016, an international task force of the Society of Critical Care Medicine (SCCM) and the European Society of Intensive Care Medicine (ESICM) published new guidelines providing definitions and diagnostic criteria for sepsis (Sepsis-3), using the Sequential Organ Failure Assessment (SOFA) score. ${ }^{1}$

Among infectious patients, a SOFA score $\geq 2$ points is considered sufficient for the diagnosis of sepsis, and is associated with an in-hospital mortality of $10 \%$. The SOFA score consists of six items, each representing one organ system: including the respiratory (partial pressure of oxygen/fraction of inspired oxygen ratio, PF ratio), cardiovascular (mean arterial pressure [MAP] and use of ionotropic/vasopressor agents), coagulation (platelet count), liver (bilirubin), kidney (urine output and creatinine), and central nervous (Glasgow Coma Scale, GCS) systems.

When treating patients, some of the tests that are used to determine the SOFA score, such as GCS and MAP, can be done at the bedside. The PF ratio requires arterial blood, but can be determined within a few minutes if point-of-care testing is available. Other tests that require more complex processes, including blood sampling, sample transportation, and laboratory processing, and thus take longer to be determined. Thus, from a physician's perspective, sepsis can be divided into two categories: early-identified (EI) sepsis which can be determined early using bedside variables, and late-identified (LI) sepsis which is determined after all SOFA items are available.

If the SOFA score had internal validity, subgroups based on criteria unrelated to the severity-time to diagnosis of the sepsis in this case, should have similar mortality. Sepsis is regarded as a medical emergency, because it is associated with a high mortality rate (10\%). If mortality rates were shown to be disproportional between patients with $\mathrm{El}$ and $\mathrm{LI}$ sepsis, this would raise concerns about the internal validity of the SOFA score. The aim of the study was to compare the mortality rates of patients with El sepsis and LI sepsis.

\section{METHODS}

\section{Study design and setting}

The study was based on a retrospective chart review. It was approved by the institutional review board of the Chonbuk National University Hospital (2018-11-001) and was given a waiver of the requirement to obtain informed consent from the patients in the study. The study was performed in a 1,200-bed urban, academic, tertiary-care university hospital. We referred to the Standards for the Reporting of Diagnostic Accuracy (STARD) recommendations when analyzing the results. ${ }^{2,3}$

\section{Patient selection}

The charts of adult patients (aged 18 years or older) admitted to the emergency department (ED) between November 1, 2016 and December 31, 2016 were screened. The senior emergency medicine resident reviewed the patient's International Statistical Classification of Diseases and Related Health Problems 10th edition (ICD-10) code at the time of their discharge. This resident was blinded to the study's objectives and outcomes. Before reviewing the medical charts, the resident was trained to select patients who met a diagnosis of infection according to the ICD-10 classification. To ensure quality control, one of researchers reviewed the data of a randomly-selected $20 \%$ sample of the charts of patients that the resident identified as meeting the criteria for inclusion in the review. Based on the Sepsis-3 guidelines, in order to be eligible for inclusion patients were required to have a diagnosis of sepsis based on a SOFA score or an acute change in their SOFA score (if available). Patients diagnosed with sepsis, who did not meet the SOFA score criteria, were excluded. Urine output was not considered in the calculation of the SOFA scores. Patients diagnosed with sepsis prior to admission were also excluded.

\section{Measurements and data collection}

Patients with a SOFA score $\geq 2$, based on their GCS, MAP and PF ratio results alone, measured within an hour of admission, are considered to have El sepsis. Patients with a SOFA score of $<2$ based on their GCS, MAP, and PF ratio results, and a total SOFA score of $\geq 2$ after their coagulation, liver, and kidney test results have been taken into account are considered to have LI sepsis.

Patients' data were collected from electronic charts by the senior emergency medicine resident, using a structured form according to the recommendations of Gilbert et al. ${ }^{4}$ The following data were collected: age, sex, emergency medical service use, referral from another hospital or facility, acuity assigned at triage (measured on a scale of 1 to 5 , according to the Korean Triage and Acuity Scale); Charlson Comorbidity Index (CCI), (hypertension, diabetes mellitus, malignancy, previous occurrence of cerebrovascular accident, dementia, chronic kidney disease, chronic liver disease, previous myocardial infarction, chronic obstructive pulmonary disease, previous peptic ulcer disease, chronic heart failure, connective tissue disease, hemiplegia, and peripheral vascular disease), source of infection (respiratory, liver-gall bladderpancreas [LGP], genitourinary, gastrointestinal, skin and skeletal, head and neck, vessel or catheter, other source, or unknown source), systolic blood pressure, diastolic blood pressure, pulse rate, respiratory rate, body temperature, $\mathrm{PO}_{2}$, lactate, platelet count, total serum levels of bilirubin, creatinine, ED length of stay, hospital length of stay, quick SOFA (qSOFA) score, SOFA score, 
Acute Physiology and Chronic Health Evaluation (APACHE) II score, progression to septic shock within 6 hours, admission to the intensive care unit (ICU), admission to a general ward, and inhospital death. The CCI was used to adjust mortality among patient with sepsis.

The laboratory test results needed to determine the SOFA score at the time of admission were also recorded. These results included mental status, partial pressure of oxygen, fraction of inspired oxygen, and use and dosage of inotropic or vasopressor agents. Records of patients' daily urine output were not available. In patients with pre-existing organ dysfunction, an acute change in the SOFA score made them eligible for inclusion if they met the diagnostic criteria for a diagnosis of sepsis. Diagnoses of sepsis and septic shock were based on the most recent international consensus (Sepsis-3). ${ }^{1}$

\section{Outcome measures}

The primary outcome measure was in-hospital mortality. Secondary measures: SOFA score, APACHE II score, septic shock within 6 hours, and ICU admission, were used as indicators of severity.

\section{Statistical analysis}

For continuous variables, the means and standard deviations were determined if the data were normally distributed, or the medians and interquartile ranges were determined if the data were not normally distributed. For discrete variables, the frequencies and percentages were determined. As the study was exploratory in nature, we did not perform any sample size calculations.

For missing information, discrete variables were treated as dummy variables and continuous variables were imputed as the mean or median values of each variable. Thus, all subjects were included in the final multivariate analysis.

In order to assess the statistical significance when making comparisons, the Student's t-test was used for normally-distributed continuous data; the Mann-Whitney U-test was used for continuous data that were not normally distributed; and the Pearson chi-square test was used for categorical data. The Fischer exact test was used instead of the chi square test for $2 \times 2$ tables if the number of observations in any of the cells was small. Two-tailed P-values $<0.05$ were considered to be statistically significant.

The mortality rates associated with the scores for each organ system in the SOFA score in this study were displayed. The mortality rates in the original study were also referenced and displayed. ${ }^{5}$

All analyses were performed using $\mathrm{R}$ ver. 3.3.1 (R Core Team, Vienna, Austria) or SAS ver. 9.1 (SAS Institute Inc., Cary, NC, USA).

\section{RESULTS}

During the study period, 4,791 adult patients visited our hospital ED. After excluding 945 patients with non-medical conditions, and 3,171 patients without infection, 675 patients were identified as potentially eligible. Among these patients, we excluded

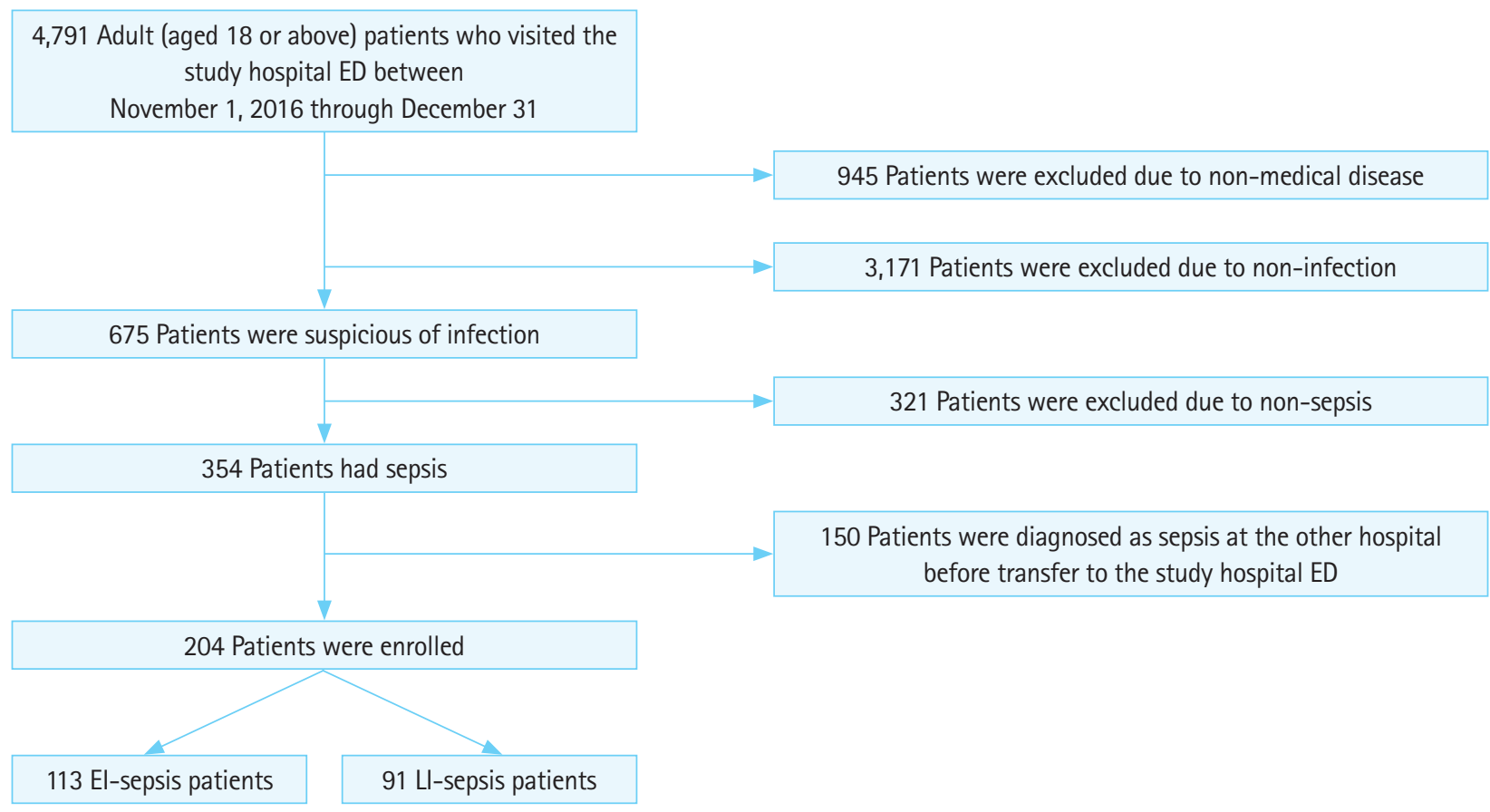

Fig. 1. Standards for the Reporting of Diagnostic Accuracy (STARD) flow diagram. ED, emergency department; El, early-identified; LI, late-identified. 
Woon Jee, et al.

Table 1. Baseline characteristics of enrolled patients

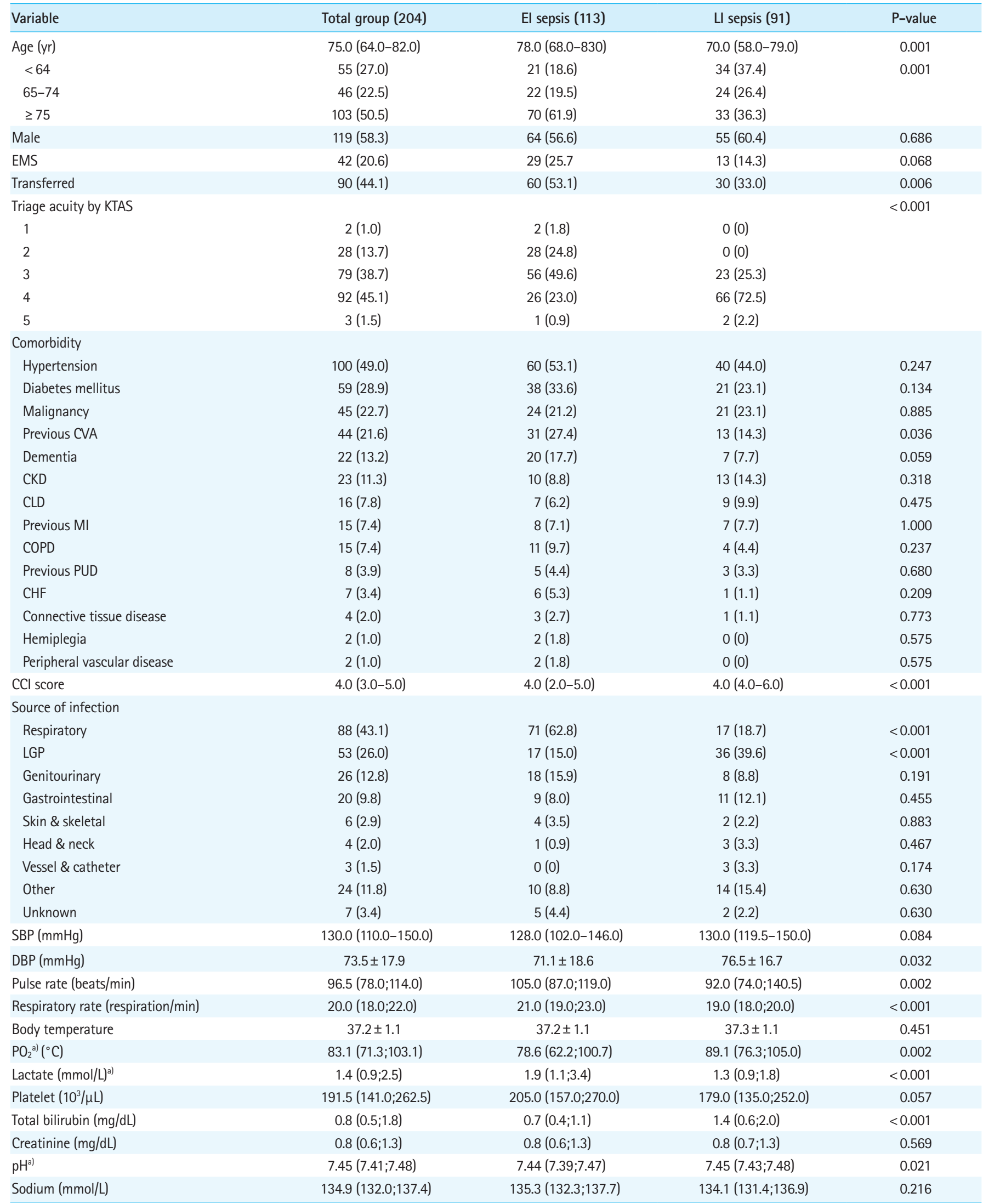


Table 1. Continued

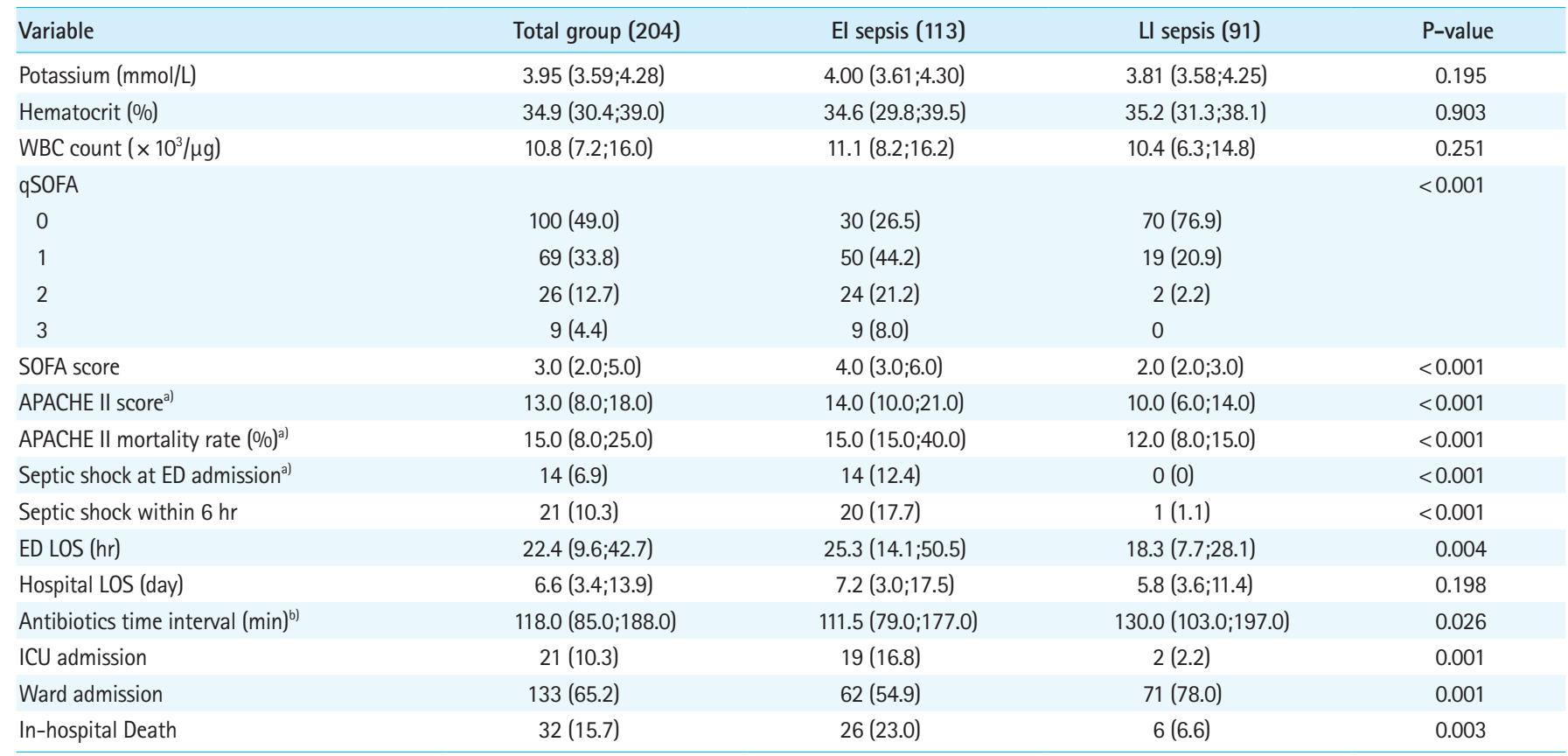

Values are presented as median (interquartile range), number (\%), or mean \pm standard deviation.

EI, early-identified; LI, late-identified; EMS, emergency medical service; KTAS, Korean triage and acuity scale; CVA, cerebrovascular accident; CKD, chronic kidney disease; CLD, chronic liver disease; MI, myocardial infarction; COPD, chronic obstructive pulmonary disease; PUD, peptic ulcer disease; CHF, congestive heart failure; CCI, Charlson Comorbidity Index; LGP, liver gall bladder pancreas; ICD-10, International Statistical Classification of Diseases and Related Health Problems 10th edition; SBP, systolic blood pressure; DBP, diastolic blood pressure; WBC, white blood cell; qSOFA, quick Sequential Organ Failure Assessment; SOFA, Sequential Organ Failure Assessment; APACHE, Acute Physiology and Chronic Health Evaluation; ED, emergency department; LOS, length of stay; ICU, intensive care unit.

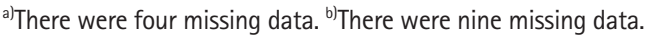

321 patients who were not diagnosed with sepsis. Of the 354 remaining patients, 150 patients were excluded because they had been diagnosed with sepsis before admission to the ED. The final sample comprised 204 patients (Fig. 1).

Table 1 shows the characteristics of the enrolled patients. Their median age was 75.0 years (interquartile range [IQR], 84.0-82.0 years), and 119 (58.3\%) were males. Hypertension (49.0\%) was the most frequent comorbidity, followed by diabetes mellitus (28.9\%), malignancy $(22.7 \%)$, and previous cerebrovascular accident (21.6\%). The median CCI was $4($ IOR, 3-5). The respiratory tract was the most frequent source of infection (43.1\%), followed by LGP $(26.0 \%)$, genitourinary $(12.8 \%)$, and gastrointestinal $(9.8 \%)$ tracts. The median serum lactate level was $1.4 \mathrm{nmol} / \mathrm{L}$ (IQR, $0.9-2.5 \mathrm{nmol} / \mathrm{L})$, and the median SOFA score was 3 (IQR, 2-5). Twenty-one patients (10.3\%) developed septic shock within six hours of admission to the ED, and were transferred to the ICU, and 32 patients (15.7\%) died during the study period.

Of the 204 patients included in the study, 113 were classified as having El sepsis, and 91 were classified as having LI sepsis. Patients with El sepsis were older, triaged with higher acuity, and had a higher median $\mathrm{CCl}$. Respiratory tract infection was more frequent in patients with El sepsis than in those with LI sepsis (39.6\% vs. $15.0 \%, P<0.001)$, while LGP infection was more frequent in patients with $\mathrm{LI}$ sepsis than in patients with El sepsis (62.8\% vs. $18.7 \%, \mathrm{P}<0.001)$. Patients with El sepsis had higher lactate levels and lower partial pressures of oxygen and higher platelet counts, while those with LI sepsis had a higher bilirubin level. Patients with El sepsis had higher qSOFA scores than patients with LI sepsis. The median SOFA score was higher in patients with El sepsis than in those with $\mathrm{LI}$ sepsis (4 [IOR, 3-6] vs. 2 [IOR, 2-3], $\mathrm{P}<0.001)$. The median APACHE II score was significantly higher in patients with El sepsis than in those with $\mathrm{LI}$ sepsis (14 [IOR, $10-21]$ vs. 10 [IOR, 6-14], $P<0.001)$. Compared to patients with $\mathrm{LI}$ sepsis, those with El sepsis had higher rates of progression to septic shock within six hours (17.7\% vs. $1.1 \%, P<0.001)$, admission to the ICU ( $16.8 \%$ vs. $2.2 \%, P=0.001)$, and mortality (23.0\% vs. $6.6 \%, P=0.003$ ) (Fig. 2 and Fig. 3).

The values of the scores representing each organ system are shown in Table 2. Higher scores were more frequent at central nerve system, cardiovascular, and respiratory scores in patients with El sepsis, while higher scores were more frequent at liver score in patients with $\mathrm{LI}$ sepsis. The time to determine the result of the respiratory score was shorter in patients with El sepsis than in those with LI sepsis (24 minutes [IOR, 17-32 minutes] vs. 32 

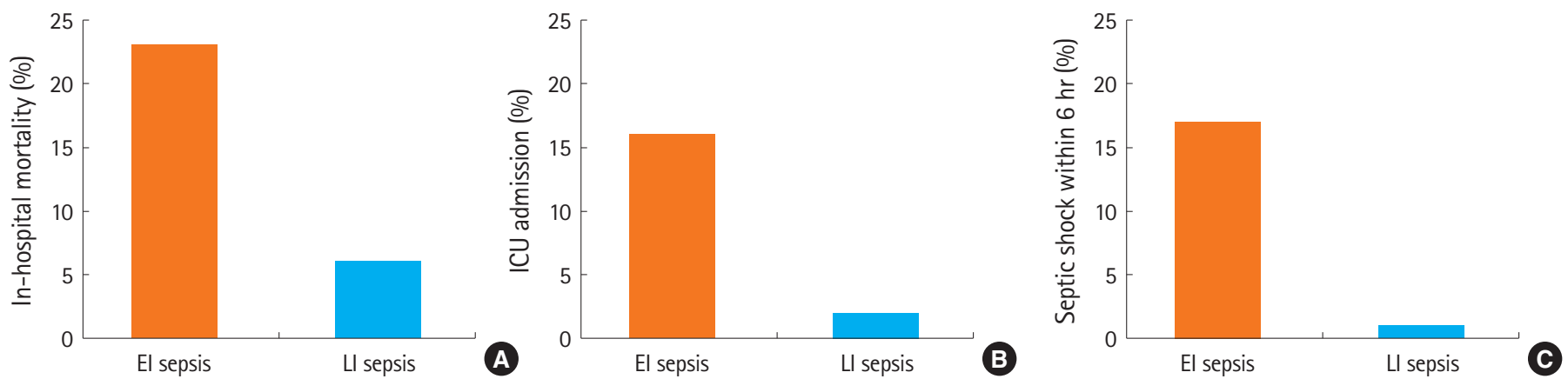

Fig. 2. Comparison of the in-hospital mortality rate (A), ICU admission rates (B), incidence of septic shock in 6 hours (C) among patients with earlyidentified sepsis (El sepsis) and late-identified sepsis (LI sepsis).
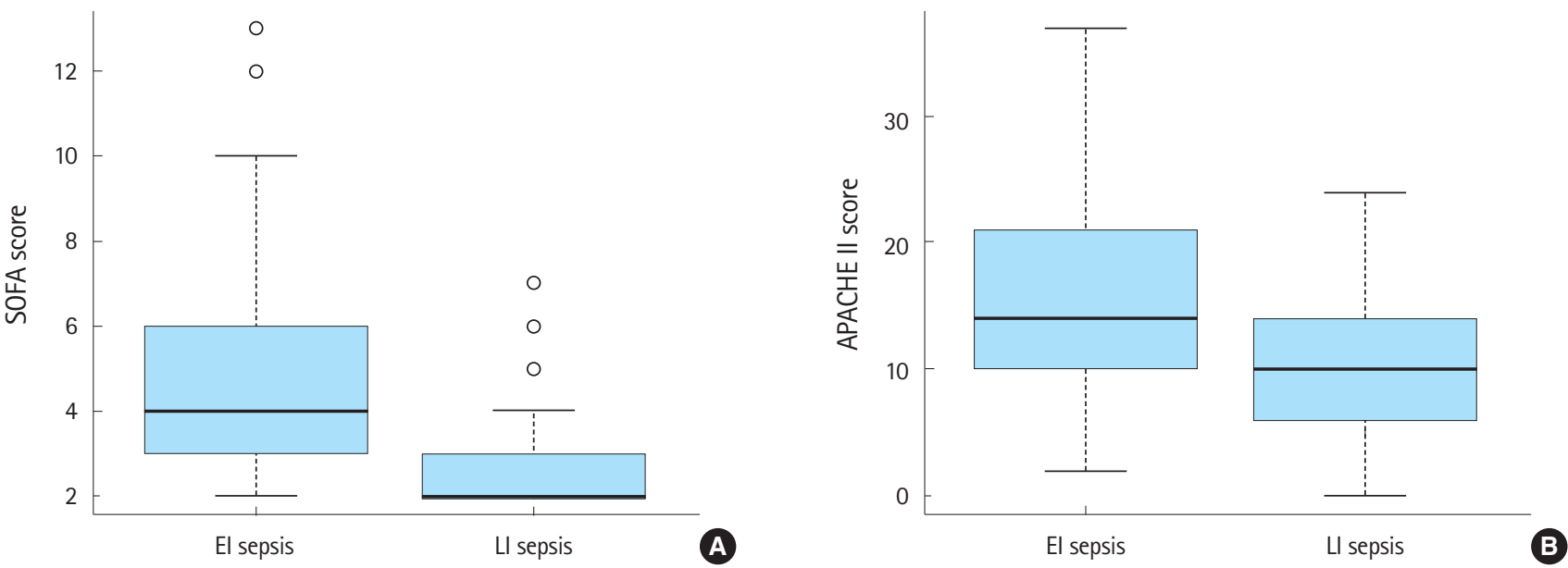

Fig. 3. Median and interquartile range of SOFA score (A) and APACHE II score (B) among patients with early-identified sepsis (El sepsis) and late-identified sepsis (LI sepsis).

minutes [IQR, 22.5-41 minutes], $\mathrm{P}<0.001)$. There was no difference between the patients with El sepsis and those with $\mathrm{LI}$ sepsis regarding the median time to determine the results of the coagulation score (67 minutes [IQR, 51-86 minutes] vs. 75 minutes [IQR, 58.5-91 minutes], $P=0.079$ ), and liver/kidney score (111 minutes [IOR, 94-157 minutes] vs. 121 minutes [IQR, 95-147.5 minutes], $\mathrm{P}=0.800)$.

The mortality rates associated with the scores for each organ system in the SOFA score, both in the original study and in this study, are shown in Fig. 4. As notable mortality gaps were found in all the scores, (especially the coagulation, hepatic, and renal scores), and the number of deaths was not recorded in the study, we did not make any statistical comparisons between the two studies.

\section{DISCUSSION}

This study showed a significant difference in the mortality rates between patients with El sepsis and those with LI sepsis. This suggests that these two conditions are not equally severe, considering the differences in the SOFA score, the rates of progression to septic shock, and the rates of ICU admission between the two groups. The low mortality rate among patients with $\mathrm{LI}$ sepsis raises a concern about the internal validity of the SOFA score system in our cohort. This is in keeping with the latest international consensus that has addressed sepsis as a medical emergency issue, based on the high mortality rates among patients with sepsis. ${ }^{1}$ The calculation of the scores representing each organ system in the SOFA score may need adjustment in order to provide better internal validity.

Sepsis is an established term, but the concept and diagnostic criteria for sepsis have been continually reviewed. The American College of Chest Physicians/SCCM consensus conference, held in 1991, defined sepsis as the systemic response to infection, manifest by two or more of the following conditions: (1) body temperature $>38^{\circ} \mathrm{C}$ or $<36^{\circ} \mathrm{C}$; (2) heart rate $>90$ beats per minute; (3) respiratory rate $>20$ breaths per minute or $\mathrm{PaCO} 2<32 \mathrm{mmHg}$; and (4) a white blood cell count $>12,000 / \mathrm{cu} \mathrm{mm,}<4,000 / \mathrm{cu} \mathrm{mm}$, 
Table 2. Distribution of the scores in each organ system

\begin{tabular}{|c|c|c|c|c|}
\hline Variable & Total group (204) & El sepsis (113) & LI sepsis (91) & P-value \\
\hline Central nervous system score & & & & $<0.001$ \\
\hline 0 & $155(76.0)$ & $66(58.4)$ & 89 (97.8) & \\
\hline 1 & $18(8.8)$ & $16(14.2)$ & $2(2.2)$ & \\
\hline 2 & $18(8.8)$ & 18 (15.9) & $0(0)$ & \\
\hline 3 & $11(5.4)$ & $11(9.7)$ & $0(0)$ & \\
\hline 4 & $2(1.0)$ & $2(1.8)$ & $0(0)$ & \\
\hline Cardiovascular score & & & & 0.009 \\
\hline 0 & $178(87.3)$ & $90(79.6)$ & 88 (96.7) & \\
\hline 1 & $21(10.3)$ & 18 (15.9) & $3(3.3)$ & \\
\hline 2 & $1(0.5)$ & $1(0.9)$ & $0(0)$ & \\
\hline 3 & $2(1.0)$ & $2(1.8)$ & $0(0)$ & \\
\hline 4 & $2(1.0)$ & $2(1.8)$ & $0(0)$ & \\
\hline Respiration score & & & & $<0.001$ \\
\hline 0 & $47(23.0)$ & $7(6.2)$ & $40(44.0)$ & \\
\hline 1 & $61(29.9)$ & $10(8.8)$ & $51(56.0)$ & \\
\hline 2 & $64(31.4)$ & $64(56.6)$ & $0(0)$ & \\
\hline 3 & $16(7.8)$ & $16(14.2)$ & $0(0)$ & \\
\hline 4 & $16(7.8)$ & $16(14.2)$ & $0(0)$ & \\
\hline Time to result (min) (missing in 4) & $28.0[19.5 ; 38.0]$ & $24.0[17.0 ; 32.0]$ & $32.0[22.5 ; 41.0]$ & $<0.001$ \\
\hline Coagulation score & & & & 0.153 \\
\hline 0 & $145(71.1)$ & $87(77.0)$ & $58(63.7)$ & \\
\hline 1 & $35(17.2)$ & $14(12.4)$ & $21(23.1)$ & \\
\hline 2 & $21(10.3)$ & $11(9.7)$ & $10(11.0)$ & \\
\hline 3 & $3(1.5)$ & $1(0.9)$ & $2(2.2)$ & \\
\hline 4 & $0(0)$ & $0(0)$ & $0(0)$ & \\
\hline Time to result (min) & $69.5[53.5 ; 89.5]$ & $67.0[51.0 ; 86.0]$ & $75.0[58.5 ; 91.0]$ & 0.079 \\
\hline Liver score & & & & $<0.001$ \\
\hline 0 & $125(61.3)$ & $87(77.0)$ & $38(41.8)$ & \\
\hline 1 & $41(20.1)$ & $11(9.7)$ & $30(33.0)$ & \\
\hline 2 & $32(15.7)$ & $12(10.6)$ & $20(22.0)$ & \\
\hline 3 & $5(2.5)$ & $3(2.7)$ & $2(2.2)$ & \\
\hline 4 & $1(0.5)$ & $0(0)$ & $1(1.1)$ & \\
\hline Time to result (min) & $118.0[94.0 ; 150.5]$ & $111.0[94.0 ; 157.0]$ & $121.0[96.0 ; 147.5]$ & 0.800 \\
\hline Kidney score & & & & 0.899 \\
\hline 0 & $141(69.1)$ & $81(71.7)$ & $60(65.9)$ & \\
\hline 1 & $35(17.2)$ & $18(15.9)$ & $17(18.7)$ & \\
\hline 2 & $13(6.4)$ & $6(5.3)$ & $7(7.7)$ & \\
\hline 3 & $10(4.9)$ & $5(4.4)$ & $5(5.5)$ & \\
\hline 4 & $5(2.5)$ & $3(2.7)$ & $2(2.2)$ & \\
\hline Time to result (min) & $118.0[94.0 ; 150.5]$ & $111.0[94.0 ; 157.0]$ & $121.0[96.0 ; 147.5]$ & 0.800 \\
\hline
\end{tabular}

Values are presented as number (\%) or median (interquartile range).

El, early-identified; LI, late-identified.

or a proportion of immature (band) forms of $>10 \% .{ }^{6}$ In 2001, a task force, composed of representatives from the SCCM, ESCIM, American College of Chest Physicians, American Thoracic Society, and Surgical Infection Society, defined sepsis as a clinical syndrome characterized by the presence of both infection and a systemic inflammatory response, and specified that the diagnosis of sepsis required the presence of general, inflammatory, hemodynamic, organ dysfunction, and tissue perfusion, variables (a total of 24 variables). ${ }^{7}$ In 2008, the Surviving Sepsis Campaign (SSC) developed a similar definition, that specified that sepsis was an "infection plus systemic manifestations of infection." ${ }^{8}$ In 2015, the SCCM/ESICM consensus (Sepsis-3), developed a definition, that defined sepsis as a life-threatening organ dysfunction caused by a dysregulated host response to infection. ${ }^{1}$ This new definition emphasized the primary role of the non-homeostatic host response to infection, the potential mortality, and the need for urgent rec- 


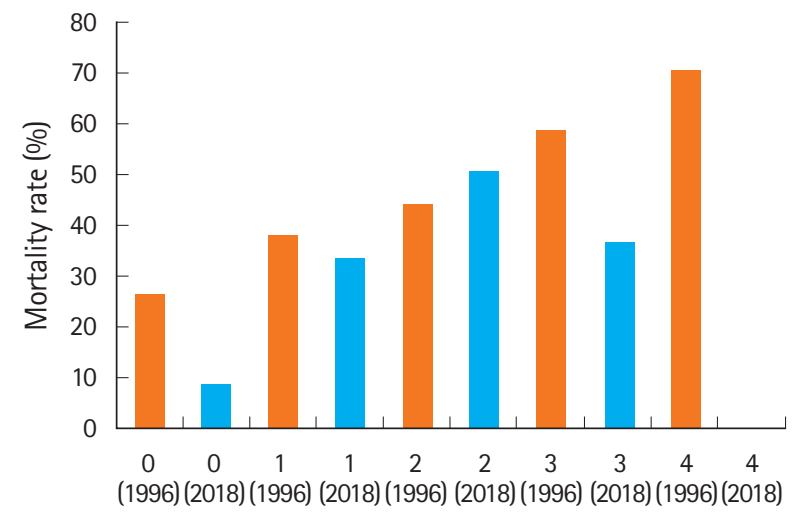

SOFA sub-score
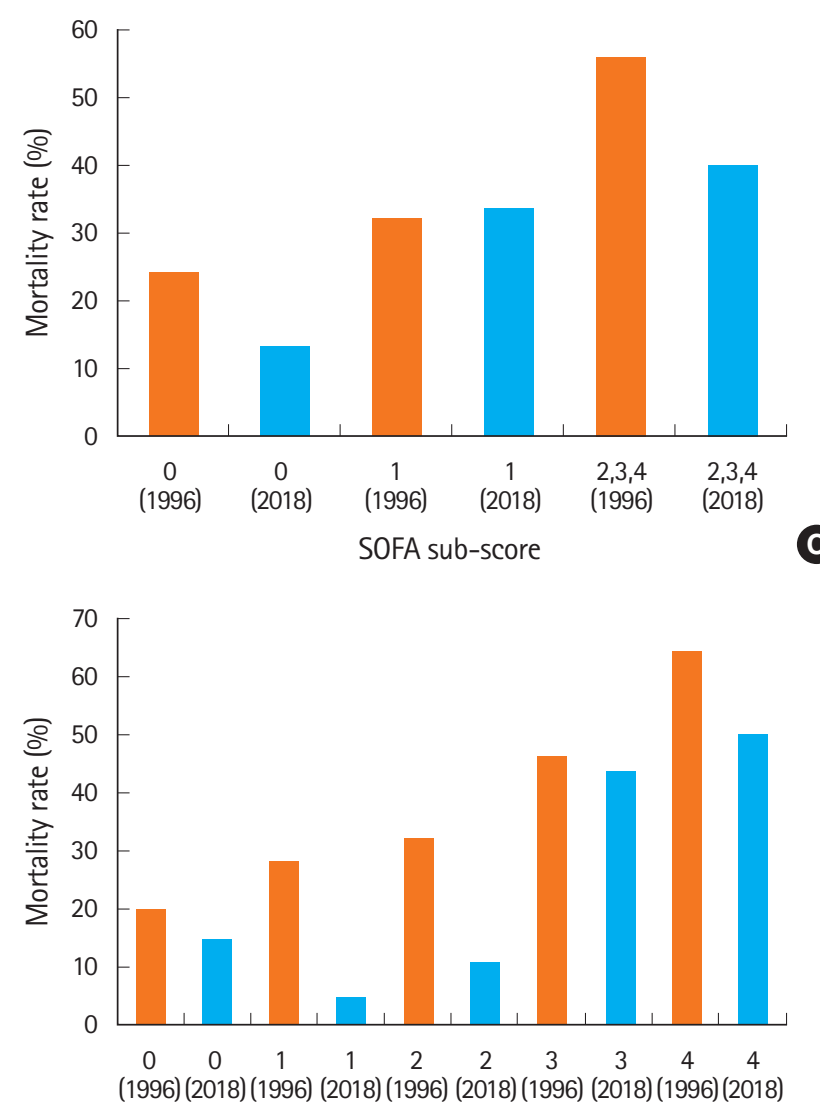

SOFA sub-score

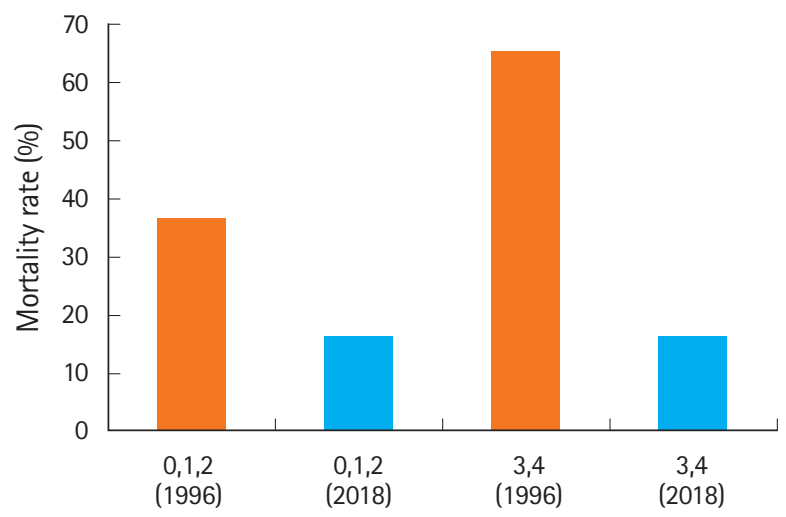

SOFA sub-score

B

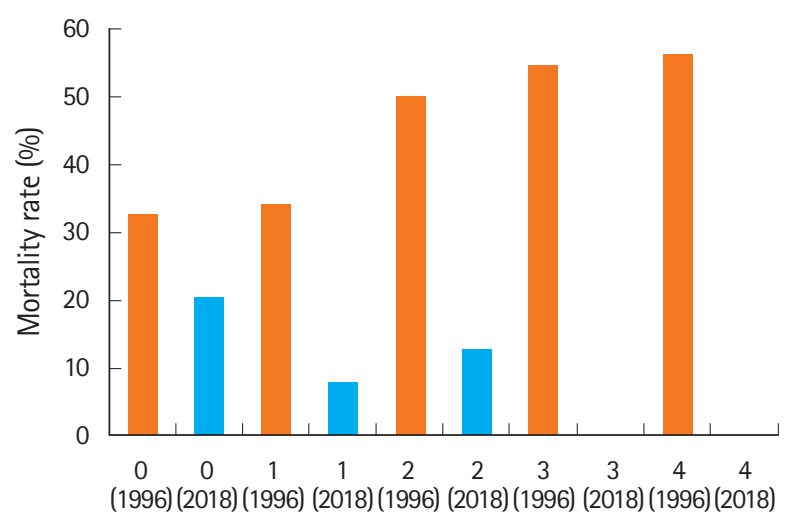

SOFA sub-score

D

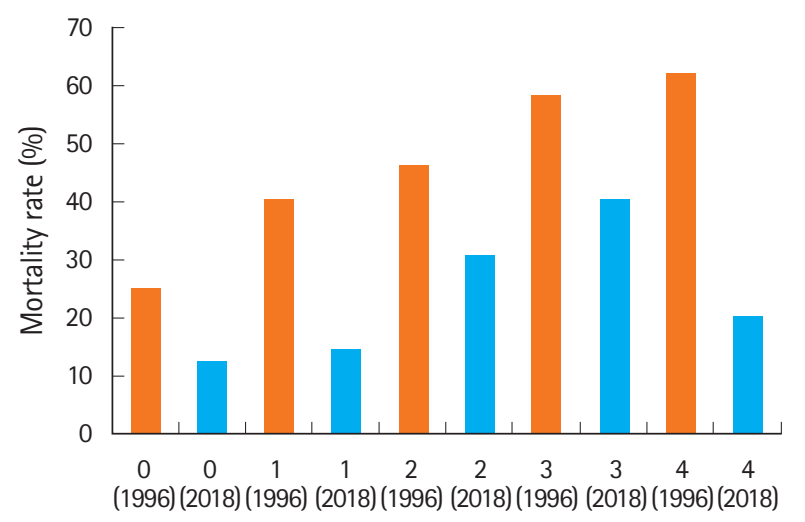

SOFA sub-score

Fig. 4. The mortality rates according to the Sequential Organ Failure Assessment (SOFA) sub-scores for each organ system in the original study and in this study. (A) Central nervous system, (B) coagulation, (C) cardiovascular, (D) liver, (E) respiratory, and (F) kidney.

ognition of sepsis. The task force recommended changing the diagnostic criteria to a baseline SOFA score of $\geq 2$ points in order to characterize organ dysfunction. The task force recommended that the baseline SOFA score should be assumed to be 0 , unless the patient was known to have preexisting (acute or chronic) organ dysfunction before the onset of infection. ${ }^{1}$ Patients in a general hospital, with presumed infection and a SOFA score $\geq 2$, have an overall mortality of approximately $10 \%{ }^{9}$
Originally, the SOFA score, developed by an ESICM consensus in 1994, was designed to quantitatively and objectively describe the degree of organ dysfunction. ${ }^{5}$ Those who developed the consensus agreed on three major points: (1) to limit the number of organ systems studied to six, (2) to use a score ranging from 0 (normal) to 4 (most abnormal) for each organ system, and (3) to record the highest values for each organ system on each day.

Further details regarding the process of score assignment to 
each organ system were not documented in the original study. ${ }^{5}$ However, Fig. 4 shows a relatively similar distribution of the mortality rates according to the score assigned to each organ system. Regarding the respiratory tract, the mortality rate was 20\% if the score was $0,26 \%$ if the score was $1,32 \%$ if the score was 2 , $46 \%$ if the score was 3, and 64\% if the score was 4 . Regarding the CNS, the mortality rate was 26\% if the score was 0,38\% if the score was 1,44\% if the score was 2,58\% if the score was 3 , and $70 \%$ if the score was 4 . Regarding the hepatic system the mortality rate was $32 \%$ if the score was $0,34 \%$ if the score was $1,50 \%$ if the score was 2, 54\% if the score was 3, and $56 \%$ if the score was 4 . Regarding the renal system, the mortality rate was $25 \%$ if the score was $0,40 \%$ if the score was $1,46 \%$ if the score was $2,58 \%$ if the score was 3 , and $62 \%$ if the score was 4 . Regarding the coagulation system, the mortality rate was $36 \%$, if the score was 0 to 2; and 65\%, if the score was 3 or 4 . Regarding the cardiovascular system, the mortality rate was approximately $24 \%$ if the score was $0,32 \%$ if the score was 1 , and $56 \%$ if the score was 2 to 4 (Fig. 4).

Many studies have validated the SOFA score in a clinical setting. ${ }^{10-12}$ After the Third International Conference Definitions for Sepsis and Septic Shock (Sepsis-3) was launched, the SOFA score remained pertinent, and was reported to be a useful predictor on its own, and superior for prognostication than other scores. ${ }^{13-15}$ Notably, people responsible for managing patients with sepsis, including physicians, researchers, and policy makers, widely agree that SOFA provides a functional score for the management of sepsis. Nevertheless, the internal validity of the SOFA score should be questioned. Firstly, the previous process to determine the score for each organ system is not well explained. Secondly, the SOFA score was designed more than 20 years ago. Because the treatment of organ dysfunction or failure has significantly improved over the past 20 years, the mortality rate is also likely to have changed. Revisions to the SOFA scoring system may be necessary in order to maintain its internal validity, especially considering that sepsis needs to be treated as a medical emergency due to the high sepsis-related mortality rate. The results of this study are noteworthy because they provide information on the internal validity of the SOFA scoring system.

The classification of sepsis into two categories (El and LI sepsis) provides a physician-centered perspective. Because half of the clinical variables that are used to calculate the SOFA score cannot be determined immediately, and because of the necessity of early diagnosis and prompt treatment of sepsis, physicians may not feel comfortable with relying on the SOFA score to make decisions regarding patient management. ${ }^{16}$ For example, ST segment elevation myocardial infarction is readily diagnosed by electrocardiography, and the need for a prompt treatment can be determined rapidly without assessing the patient's SOFA score. In contrast to this, some infected patients may not have early signs and symptoms of sepsis, making it difficult to make prompt decisions regarding the initiation of appropriate treatment. Of note, qSOFA seems to be insufficient in this cohort, considering that $17.1 \%$ of the patients in the study had qSOFA scores $\geq 2(29.2 \%$ in those with El sepsis and 2.2\% in those with LI sepsis). Furthermore, the SSC recommends the implementation of the SSC Hour1 Bundle, a set of decision points to be taken within the first hour of sepsis recognition. The bundle comprises the measurement of serum lactate levels, blood culture, administration of broad-spectrum antibiotics, administration of crystalloid for hypotension or hyperlactatemia, followed by the administration of vasopressors. ${ }^{16}$ However, this study shows that the SSC Hour-1 Bundle has limitations because it took approximately two hours to diagnose $\mathrm{LI}$ sepsis.

Sepsis severity was found to be lower in patients with LI sepsis, as shown by the lower rates of mortality, septic shock and ICU admission, as well as the lower median SOFA score in this group. This can help minimize the stress among physicians regarding the need for early diagnosis and treatment for $\mathrm{LI}$ sepsis. On the other hand, concerns may be raised about the internal validity of the SOFA score. In order to achieve internal validity, it may be necessary to adjust the SOFA scores for each organ system. As shown in Fig. 4, notable differences in mortality according to each score were found when the original study was compared to this study, especially a temporal decrease in the mortality rates associated with the scores representing the hepatic and renal systems. The decrease in mortality rates over time may be due to improved radiologic and endoscopic interventions, such as percutaneous transhepatic biliary drainage, percutaneous transhepatic gallbladder drainage, and endoscopic retrograde biliary drainage. ${ }^{17-19}$ Other factors, such as the introduction of technologies for continuous renal replacement therapy ${ }^{20,21}$ and early goal directed therapy for treatment of severe sepsis ${ }^{22}$ may also play a role. In this study, there were no deaths among the patients with the highest SOFA scores of the CNS, coagulation system, and hepatic system. This may be attributable to the small number of patients in these categories.

The main limitations of this study was its retrospective design, using a simple chart review as the source of data, and carrying out the study at only one facility. These limitations limit the inferences that can be drawn from the study results. A multicenter study is required to better evaluate these data. However, our results confirm that mortality rates differ significantly among patients with El sepsis and LI sepsis, and that the internal validity of 
the SOFA score needs to be reevaluated. Most of the data required for the analysis were easy to obtain, and can be considered to be reliable. Secondly, the approximate difference of two hours in the time taken to diagnose El sepsis and LI sepsis is longer than that which occurs in some clinical settings, thus limiting the generalizability of our findings. For example, serum platelet count, and serum bilirubin and creatinine levels, can be rapidly determined using commercial point-of-care testing devices. ${ }^{23-25}$ This, narrows the division of El sepsis and $\mathrm{LI}$ sepsis and thus makes the distinction between El sepsis and $\mathrm{LI}$ sepsis less useful from a physician perspective. Nevertheless, the disproportionately high mortality rate in patients with El sepsis compared to those with $\mathrm{LI}$ sepsis is still noteworthy. Thirdly, alternative criteria such a relying on single components of the SOFA score (e.g., the cardiovascular system or respiratory system sub-scores) could be useful in dividing patients with sepsis into these two subgroups for the purpose of clinical management. However, a disadvantage of using subscores is that it could lead to the development of many fragmented criteria. Therefore, we chose to study sepsis from a physician-centered perspective, in order to facilitate physicians' bedside decision-making regarding treatment.

In conclusion, the mortality rates were significantly higher among patients with El sepsis than in those with LI sepsis. Considering that the results of tests performed at the bedside had a greater prognostic value than tests with a longer turn-around time, we recommend that the SOFA scoring system be revised.

\section{CONFLICT OF INTEREST}

No potential conflict of interest relevant to this article was reported.

\section{REFERENCES}

1. Singer M, Deutschman CS, Seymour CW, et al. The third international consensus definitions for sepsis and septic shock (Sepsis-3). JAMA 2016;315:801-10.

2. Bossuyt PM, Reitsma JB, Bruns DE, et al. Towards complete and accurate reporting of studies of diagnostic accuracy: the STARD initiative. BMJ 2003;326:41-4.

3. Simel DL, Rennie D, Bossuyt PM. The STARD statement for reporting diagnostic accuracy studies: application to the history and physical examination. J Gen Intern Med 2008;23:768-74.

4. Gilbert EH, Lowenstein SR, Koziol-McLain J, Barta DC, Steiner J. Chart reviews in emergency medicine research: Where are the methods? Ann Emerg Med 1996;27:305-8.

5. Vincent JL, Moreno R, Takala J, et al. The SOFA (Sepsis-related
Organ Failure Assessment) score to describe organ dysfunction/failure. On behalf of the Working Group on Sepsis-Related Problems of the European Society of Intensive Care Medicine. Intensive Care Med 1996;22:707-10.

6. Bone RC, Balk RA, Cerra FB, et al. Definitions for sepsis and organ failure and guidelines for the use of innovative therapies in sepsis. The ACCP/SCCM Consensus Conference Committee. American College of Chest Physicians/Society of Critical Care Medicine. Chest 992;101:1644-55.

7. Levy MM, Fink MP, Marshall JC, et al. 2001 SCCM/ESICM/ ACCP/ATS/SIS International Sepsis Definitions Conference. Crit Care Med 2003;3:1250-6.

8. Dellinger RP, Levy MM, Carlet JM, et al. Surviving Sepsis Campaign: international guidelines for management of severe sepsis and septic shock: 2008. Crit Care Med 2008;36:296327.

9. Seymour CW, Liu VX, Iwashyna TJ, et al. Assessment of clinical criteria for Sepsis: For the Third International Consensus Definitions for Sepsis and Septic Shock (Sepsis-3). JAMA 2016; 315:762-74.

10. Vincent JL, de Mendonça $A$, Cantraine $F$, et al. Use of the SOFA score to assess the incidence of organ dysfunction/failure in intensive care units: results of a multicenter, prospective study. Working group on "sepsis-related problems" of the European Society of Intensive Care Medicine. Crit Care Med 1998;26: 1793-800.

11. Ferreira FL, Bota DP, Bross A, Melot C, Vincent JL. Serial evaluation of the SOFA score to predict outcome in critically ill patients. JAMA 2001;286:1754-8.

12. Cardenas-Turanzas M, Ensor J, Wakefield $C$, et al. Cross-validation of a Sequential Organ Failure Assessment score-based model to predict mortality in patients with cancer admitted to the intensive care unit. J Crit Care 2012;27:673-80.

13. Khwannimit B, Bhurayanontachai $R$, Vattanavanit V. Comparison of the performance of SOFA, qSOFA and SIRS for predicting mortality and organ failure among sepsis patients admitted to the intensive care unit in a middle-income country. J Crit Care 2018;44:156-60.

14. Garcia-Gigorro R, Saez-de la Fuente I, Marin Mateos H, Andres-Esteban EM, Sanchez-Izquierdo JA, Montejo-Gonzalez JC. Utility of SOFA and $\triangle$-SOFA scores for predicting outcome in critically ill patients from the emergency department. Eur J Emerg Med 2018;25:387-93.

15. Raith EP, Udy AA, Bailey $M$, et al. Prognostic accuracy of the SOFA score, SIRS criteria, and qSOFA score for in-hospital mortality among adults with suspected infection admitted to the intensive care unit. JAMA 2017;317:290-300. 
16. Levy MM, Evans LE, Rhodes A. The Surviving Sepsis Campaign Bundle: 2018 Update. Crit Care Med 2018;46:997-1000.

17. Leese T, Neoptolemos JP, Baker AR, Carr-Locke DL. Management of acute cholangitis and the impact of endoscopic sphincterotomy. Br J Surg 1986;73:988-92.

18. Boender J, Nix GA, de Ridder MA, et al. Endoscopic sphincterotomy and biliary drainage in patients with cholangitis due to common bile duct stones. Am J Gastroenterol 1995;90:233-8.

19. James PD, Kaplan GG, Myers RP, et al. Decreasing mortality from acute biliary diseases that require endoscopic retrograde cholangiopancreatography: a nationwide cohort study. Clin Gastroenterol Hepatol 2014;12:1151-9.

20. Ronco C, Bellomo R. Continuous renal replacement therapy: evolution in technology and current nomenclature. Kidney Int Suppl 1998;66:S160-4.
21. Uchino $S$, Kellum JA, Bellomo $R$, et al. Acute renal failure in critically ill patients: a multinational multicenter study. JAMA 2005;294:813-8.

22. Rivers $E$, Nguyen $B$, Havstad $S$, et al. Early goal-directed therapy in the treatment of severe sepsis and septic shock. N Engl J Med 2001;345:1368-77.

23. Weqas. POCT bilirubin [Internet]. Cardiff: Weqas [cited 2019 Apr 4]. Available from: http://www.weqas.com/services/pocteqa/poct-bilirubin/.

24. Shephard MD. Point-of-care testing and creatinine measurement. Clin Biochem Rev 2011;32:109-14.

25. Sysmex. Faster answers: earlier treatment [Internet]. Lincolnshire, IL: Sysmex [cited 2019 Apr 4]. Available from: http:// pages.sysmex.com/XW-100_Waived_CBC_landing.html. 